OPEN ACCESS

Edited by:

Jerzy Chudek,

Medical University of Silesia, Poland

Reviewed by:

Valdo Jose Dias Da Silva

Universidade Federal do Triângulo

Mineiro, Brazi

Maciej Wybraniec

School of Medicine in

Katowice, Poland

*Correspondence:

Yong Liu

liuyong@gdph.org.cn

Jianfeng Ye

yipj@@otmail.com

†These authors have contributed equally to this work

Specialty section:

This article was submitted to

General Cardiovascular Medicine,

a section of the journal

Frontiers in Cardiovascular Medicine

Received: 28 November 2021

Accepted: 03 February 2022

Published: 07 March 2022

Citation:

Zhou D, Lun Z, Wang B, Liu J, Liu L, Chen G, Ying M, Li H, Chen S, Tan N,

Chen J, Liu Y and Ye J (2022) Association Between Non-Recovered Contrast-Associated Acute Kidney Injury and Poor Prognosis in Patients Undergoing Coronary Angiography. Front. Cardiovasc. Med. 9:823829.

doi: 10.3389/fCvm.2022.823829

\section{Association Between Non-Recovered Contrast-Associated Acute Kidney Injury and Poor Prognosis in Patients Undergoing Coronary Angiography}

\author{
Dianhua Zhou ${ }^{1 \dagger}$, Zhubin Lun ${ }^{1,2,3 \dagger}$, Bo Wang ${ }^{2 \dagger}$, Jin Liu ${ }^{2 \dagger}$, Liwei Liu ${ }^{2,4}$, Guanzhong Chen ${ }^{2}$, \\ Ming Ying ${ }^{2}$, Huanqiang $\mathrm{Li}^{2}$, Shiqun Chen ${ }^{2}$, Ning Tan ${ }^{2}$, Jiyan Chen ${ }^{2}$, Yong Liu ${ }^{2 *}$ and \\ Jianfeng $\mathrm{Ye}^{1 *}$
}

\begin{abstract}
'Department of Cardiology, Dongguan TCM Hospital, Dongguan, China, ${ }^{2}$ Guangdong Provincial Key Laboratory of Coronary Heart Disease Prevention, Department of Cardiology, Guangdong Cardiovascular Institute, Guangdong Provincial People's Hospital, Guangdong Academy of Medical Sciences, Guangzhou, China, ${ }^{3}$ The First School of Clinical Medicine, Guangdong Medical University, Zhaniiang, China, ${ }^{4}$ Department of Cardiology, Zhongshan Hospital, Shanghai Institute of Cardiovascular Diseases, Fudan University, Shanghai, China
\end{abstract}

Background: Previous studies have shown that renal function recovery after acute kidney injury (AKI) was associated with decreased risk of all-cause mortality. However, little is known about the correlation between renal function recovery and long-term prognosis in patients with contrast-associated acute kidney injury (CA-AKI) undergoing coronary angiography (CAG).

Methods: We retrospectively enrolled 5,865 patients who underwent CAG. CA-AKI was defined as an increase in serum creatinine $(\mathrm{SCr}) \geq 50 \%$ or $\geq 0.3 \mathrm{mg} / \mathrm{dl}$ from baseline within $72 \mathrm{~h}$ post procedure. Recovered CA-AKI was defined as a decrease in $\mathrm{SCr}$ to baseline or no CA-AKI level. The first endpoint was long-term all-cause mortality. Kaplan-Meier analysis and Cox regression analysis were used to investigate the association between kidney function recovery and long-term mortality.

Results: During the median follow-up period of 5.25 years, the overall long-term mortality was $20.07 \%$, and the long-term mortality in patients with recovered CA-AKI and non-recovered CA-AKI was 17.46 and $27.44 \%$, respectively. After multivariate Cox hazard regression, non-recovered CA-AKI was significantly associated with long-term mortality, while recovered CA-AKI was not [recovered CA-AKI vs. no CA-AKI, hazard ratio $(H R)=1.06$, 95\% confidence interval $(C l): 0.81-1.39, p=0.661$; non-recovered CA-AKI vs. no CA-AKI, HR =1.39, 95\% Cl: 1.21-1.60, $p<0.001]$. In the subgroup of CAD, both recovered $\mathrm{CA}-\mathrm{AKI}$ and non-recovered $\mathrm{CA}-\mathrm{AKI}$ were associated with increased risk of long-term all-cause mortality. However, in other subgroup analyses, only non-recovered CA-AKI was associated with increased risk of long-term all-cause mortality.

Conclusion: Our results found that non-recovered CA-AKI is significantly associated with long-term mortality. In patients with CAD, recovered CA-AKI can still increase the risk of all-cause mortality. Clinicians need to pay more attention to patients suffering 
from CA-AKI, whose kidney function has not recovered. In addition, active prevention treatments should be taken by patients with CAD.

Keywords: recovered, non-recovered, contrast-associated acute kidney injury, coronary angiography, all-cause mortality

\section{INTRODUCTION}

Contrast-associated acute kidney injury (CA-AKI) is a common complication after coronary angiography (CAG), with an incidence of $2-20 \%$ (1). It has been known in previous studies that CA-AKI involves serious clinical prognosis, such as increased rate of rehospitalization, end-stage renal failure, and mortality (2-6). The pathogenesis of CA-AKI is mainly related to hemodynamic changes in the kidney, the toxic effect, and oxidative stress of contrast agent on renal tubular cells $(7,8)$. Endothelin, nitric oxide, and prostaglandins, which are vasoactive substances, mediate changes in vascular motility, resulting in ischemic injury (9-11). A previous study demonstrated that among patients with acute kidney injury (AKI) during hospitalization, the long-term mortality of patients with renal function recovery was lower than that of patients without renal function recovery (12). In addition, patients with recovered renal function have a lower risk of short-term mortality in patients undergoing open abdominal aortic aneurysm surgery or with dialysis-requiring $\operatorname{AKI}(13,14)$.

However, in the case of CA-AKI, few studies were conducted to explore the prognostic difference between recovery and nonrecovery of renal function. Therefore, this study aims to assess the relationship between the recovery of renal function and longterm all-cause mortality in patients with CA-AKI, undergoing coronary angiography (CAG).

\section{MATERIALS AND METHODS}

\section{Enrollment and Treatment}

This observational study was conducted using data from the Cardiorenal ImprovemeNt (CIN)-I study, which was processed in the largest cardiovascular center in South China (Guangdong Provincial People's Hospital, China; ClinicalTrials.gov NCT04407936). The baseline information, including demographics, laboratory test results, mortality, and other clinical information, were extracted from the electronic clinical management records system of the Guangdong Provincial People's Hospital from January 2007 to December 2018. Follow-up data was monitored and recorded by trained nurses through outpatient interviews and telephonic follow-up. The follow-up information of patients lost to follow-up was retrieved from Guangdong Public Security System. Senior cardiologists were responsible for data quality control and periodical data verification. All patients undergoing CAG between January 1, 2007 and December 31, 2018 were screened. During this period, 88,938 patients had undergone CAG. We excluded patients (1) <18 years old $(n=0)$; (2) who lacked serum creatinine ( $\mathrm{SCr}$ ) concentration at baseline and 1, 2, 3 days after contrast agent exposure $(n=81,892)$;
(3) with missing follow-up information on mortality $(n=$ 1,182) (Figure 1). The study was approved by the Ethics Committee of Guangdong Provincial People's Hospital (No. GDREC2019555H) and conformed to the principles outlined in the Declaration of Helsinki. CAG and percutaneous coronary intervention (PCI) were executed by clinicians based on the patient's condition and the standard clinical practice guidelines.

\section{Endpoint and Definitions}

The endpoint of our research was long-term all-cause mortality. CA-AKI was defined as an increase in $\mathrm{SCr} \geq 50 \%$ or $\geq 0.3$ $\mathrm{mg} / \mathrm{dl}$ from baseline within $72 \mathrm{~h}$ after the CAG (15). Recovered CA-AKI was defined as a decrease in serum creatinine to baseline or no CA-AKI level according to the last measurement of $\mathrm{SCr}$ within $72 \mathrm{~h}$ after CAG. Non-recovered CA-AKI was considered when SCr was not recovered within $72 \mathrm{~h}$. Coronary artery disease (CAD) was diagnosed by CAG and was depicted as $50 \%$ stenosis of at least one coronary artery. CAD was also determined according to the International Statistical Classification of Diseases and Related Health Problems 10th Revision (ICD-10). In addition, comorbidities included acute myocardial infarction (AMI), diabetes mellitus, hypertension, congestive heart failure (CHF), and chronic kidney disease (CKD). We used the Modification of Diet in Renal Disease (MDRD) equation to calculate the estimated glomerular filtration rate (eGFR), and CKD was defined as an eGFR $<60 \mathrm{ml} / \mathrm{min} / 1.73$ $\mathrm{m}^{2}(16,17)$. Anemia was defined as a baseline hematocrit value $<39 \%$ for men or $<36 \%$ for women according to the WHO criteria (18). CHF was defined as New York Heart Association (NYHA) functional class $>2$, and Killip class $>1$.

\section{Statistical Analysis}

The study population was divided into three groups, namely no CA-AKI, recovered CA-AKI, and non-recovered CA-AKI. Continuous variables were compared using one-way analysis of variance (ANOVA), and categorical data were analyzed by the Pearson chi-squared test. The cumulative mortality was determined by Kaplan-Meier (K-M) analysis, and a log-rank test was used to assess differences between curves. Univariate and multivariate Cox regression analyses were used to evaluate the association between the two types of CA-AKI and longterm all-cause mortality. We performed a subgroup analysis of patients with CAD, CKD, hypertension, CHF, and diabetes mellitus. All data analyses were performed using $\mathrm{R}$ software (version 3.6.5; R Foundation for Statistical Computing, Vienna, Austria). A two-sided $p<0.05$ indicated significance for all analyses. 


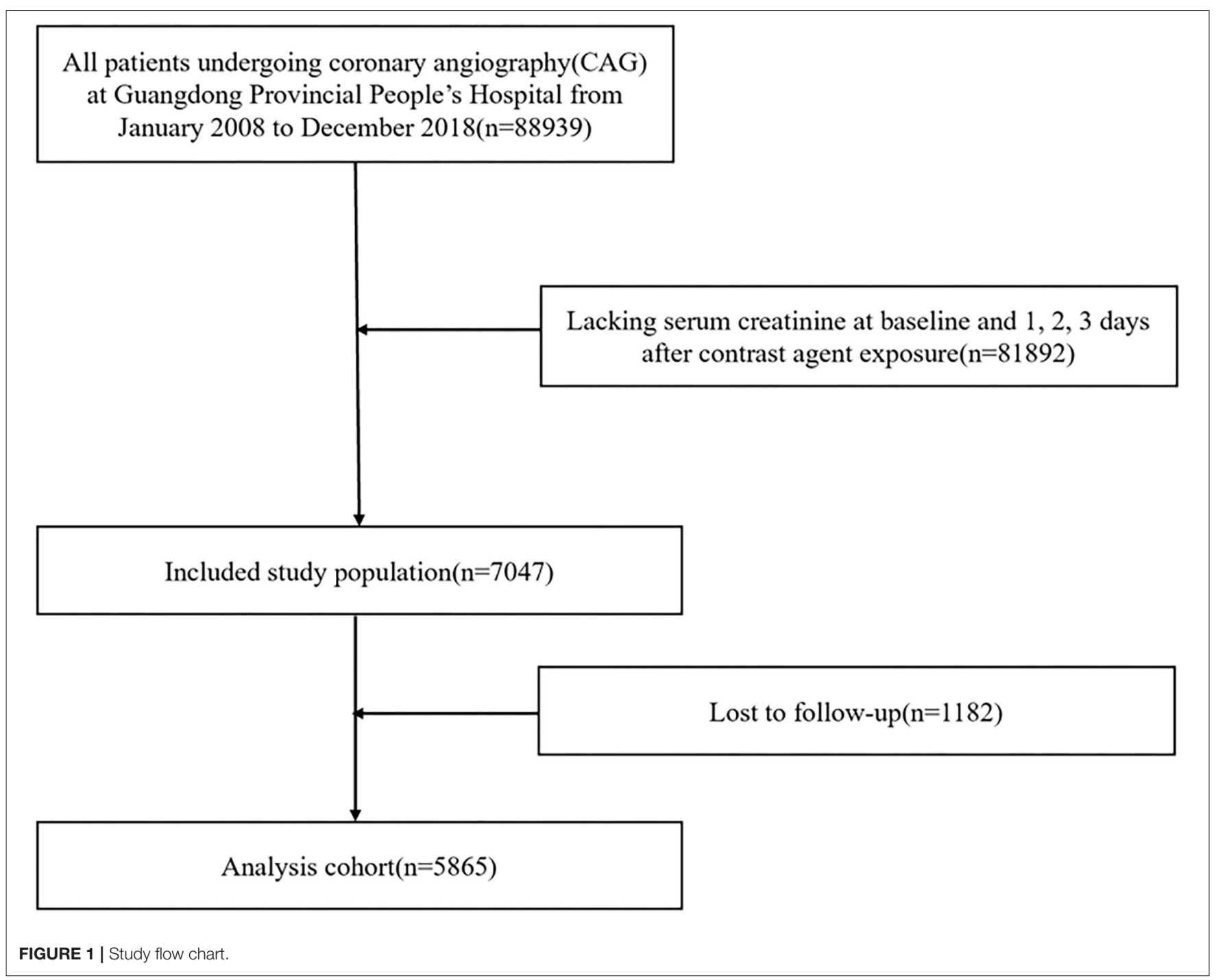

\section{RESULTS}

\section{Clinical and Procedural Characteristics}

A total of 5,865 consecutive patients who underwent CAG were enrolled in this study. All included patients were divided into three different groups: 4,401 (75.04\%) patients with no CAAKI, $338(5.76 \%)$ patients with recovered CA-AKI, and 1,126 (19.20\%) patients with non-recovered CA-AKI. The baseline clinical characteristics of all the patients are shown in Table 1. Overall, the mean age was $63.8 \pm 10.7$ years, with men accounting for $67.88 \%$ of the population. Patients diagnosed with CAD, diabetes mellitus, CHF, and CKD accounted for 67.03, 26.77, 22.11 , and $37.03 \%$, respectively.

The comorbidity rate of AMI, diabetes mellitus, PCI, and hypertension was higher in the normal group. Patients in the non-recovered CA-AKI group had lower contrast media volume as well as higher prevalence of CKD and CHF.

\section{Primary Outcomes}

During the follow-up period (mean, 5.25 years), a total of 1,177 all-cause deaths occurred. The mortality in the three groups was $18.38 \%$ for no CA-AKI (809 patients), $17.46 \%$ for recovered CA-AKI (59 patients), and $27.44 \%$ for non-recovered CA-AKI (309 patients). There were significant differences in long-term mortality among patients without CA-AKI, patients with recovered CA-AKI, and patients with non-recovered CAAKI in Kaplan-Meier curve analysis $(p<0.001)$ (Figure 2).

After adjusting for age $\geq 75$ years, sex, CKD, AMI, PCI, CHF, hypertension, diabetes mellitus, anemia, contrast media volume, and albumin, multivariate Cox regression analysis revealed that compared with no CA-AKI group, non-recovered CA-AKI group was significantly associated with increased mortality [adjusted hazard ratio (HR): 1.39, 95\% confidence interval (CI):1.21-1.60, $p<0.001$ ], while the recovered CA-AKI group was not (adjusted HR: $1.06,95 \%$ CI: $0.81-1.39, p=0.661$ ) (Figure 3). 
TABLE 1 | Baseline characteristics.

\begin{tabular}{|c|c|c|c|c|c|}
\hline Characteristic & Overall $(n=5,865)$ & No CA-AKI $(n=4,401)$ & Recovered CA-AKI $(n=338)$ & $\begin{array}{c}\text { Non-recovered } \\
\text { CA-AKI }(n=1,126)\end{array}$ & $P$-value \\
\hline Male, $n(\%)$ & $3,981(67.88)$ & 3,062 (69.58) & 214 (63.31) & 705 (62.61) & $<0.001$ \\
\hline Age, year & $63.82(10.74)$ & $63.72(10.84)$ & 63.39 (10.51) & 64.37 (10.42) & 0.146 \\
\hline Age>75 year, $n(\%)$ & 926 (15.79) & $694(15.77)$ & $47(13.91)$ & 185 (16.43) & 0.535 \\
\hline CAD, $n(\%)$ & 3,928 (67.03) & 3,199 (72.77) & $158(46.75)$ & $571(50.71)$ & $<0.001$ \\
\hline AMI, $n(\%)$ & $1,180(20.20)$ & $956(21.83)$ & 43 (12.76) & $181(16.07)$ & $<0.001$ \\
\hline $\begin{array}{l}\text { Diabetes mellitus, } \\
n(\%)\end{array}$ & $1,564(26.77)$ & 1,204 (27.49) & 74 (21.96) & $286(25.40)$ & 0.045 \\
\hline $\begin{array}{l}\text { Hypertension, } n \\
\text { (\%) }\end{array}$ & 2,966 (50.76) & 2,327 (53.13) & 145 (43.03) & $494(43.87)$ & $<0.001$ \\
\hline CKD, $n(\%)$ & $2,172(37.03)$ & 1,581 (35.92) & 102 (30.18) & 489 (43.43) & $<0.001$ \\
\hline $\mathrm{CHF}, n(\%)$ & 1,292 (22.11) & 849 (19.38) & $81(24.04)$ & $362(32.15)$ & $<0.001$ \\
\hline Anemia, $n(\%)$ & $2,389(41.00)$ & 1,733 (39.70) & 126 (37.28) & 530 (47.15) & $<0.001$ \\
\hline WBC, $10^{9} / \mathrm{L}$ & $8.31(3.14)$ & $8.37(3.16)$ & $8.01(2.92)$ & $8.20(3.12)$ & 0.055 \\
\hline $\mathrm{RBC}, 10^{12} / \mathrm{L}$ & $4.40(0.71)$ & $4.42(0.70)$ & $4.46(0.66)$ & $4.31(0.73)$ & $<0.001$ \\
\hline Albumin, g/L & $34.89(4.86)$ & 34.91 (4.73) & 35.45 (4.95) & $34.66(5.30)$ & 0.032 \\
\hline $\mathrm{MCHC}, \mathrm{g} / \mathrm{L}$ & 333.06 (12.36) & 333.41 (12.21) & 331.62 (12.87) & 332.13 (12.68) & 0.001 \\
\hline $\begin{array}{l}\text { Hemoglobin, } \\
10^{12} / \mathrm{L}\end{array}$ & 127.79 (19.79) & $128.68(19.21)$ & $128.35(20.01)$ & $124.14(21.44)$ & $<0.001$ \\
\hline Hematocrit & $0.38(0.06)$ & 0.39 (0.06) & $0.39(0.06)$ & $0.37(0.06)$ & $<0.001$ \\
\hline Cys-C, mg/L & $1.43(0.82)$ & $1.36(0.74)$ & $1.66(1.37)$ & $1.80(0.95)$ & $<0.001$ \\
\hline $\begin{array}{l}\text { eGFR, } \\
\mathrm{ml} / \mathrm{min} / 1.73 \mathrm{~m}^{2}\end{array}$ & $68.94(28.86)$ & $69.51(28.21)$ & 71.98 (29.10) & 65.80 (31.03) & $<0.001$ \\
\hline ACEl/ARB, $n(\%)$ & 1,979 (35.81) & 1,698 (40.04) & 79 (24.41) & 202 (20.95) & $<0.001$ \\
\hline Beta-blocker, $n(\%)$ & 3,717 (67.26) & 2,998 (70.69) & 187 (58.26) & 532 (55.19) & $<0.001$ \\
\hline Statin, $n(\%)$ & 3,772 (67.35) & $3,160(74.51)$ & 139 (43.30) & 423 (43.88) & $<0.001$ \\
\hline CMV, ml & 138.22 (92.19) & 144.32 (90.97) & 126.75 (109.11) & 117.79 (88.15) & $<0.001$ \\
\hline $\mathrm{PCl}, n(\%)$ & 3,110 (53.03) & 2,603 (59.15) & 119 (35.21) & 388 (37.97) & $<0.001$ \\
\hline
\end{tabular}

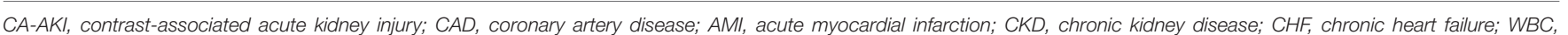

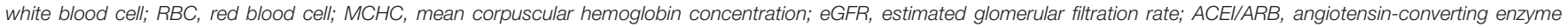
inhibitor/angiotensin receptor blocker; CMV, contrast media volume; PCl, percutaneous coronary intervention.

\section{Subgroup Analysis}

In subgroup analysis for patients complicated with CAD, both patients with recovered CA-AKI and non-recovered CA-AKI had an increased risk of long-term mortality when compared to that of patients without CA-AKI (Figure 4). In the rest of the subgroup analysis, including CKD, CHF, hypertension, and diabetes mellitus, non-recovered CA-AKI group could still increase the risk of all-cause mortality compared with no CA-AKI group. However, there was no association between the recovered CA-AKI group and long-term all-cause death.

\section{DISCUSSION}

Our results showed that patients with non-recovered CA-AKI had a worse long-term prognosis, while the patients with recovered CA-AKI did not. We found similar results in various subgroups, such as $\mathrm{CKD}$, diabetes mellitus, hypertension, and CHF groups. This study emphasized that clinicians need to pay more attention to patients with non-recovered CA-AKI in clinical practice.

According to SCr level before discharge,we divided CAAKI patients into recovered CA-AKI groups and non-recovered
CA-AKI groups. We found that the patients who recovered from CA-AKI were similar to patients without CA-AKI, based on longterm mortality. This was not consistent with what Wehbe et al. reported in their study (19). The reason could be that the lung transplant patients in the study by Wehbe et al. had more severe kidney immune damage. Similar results have been found in the study by Welten et al. (13). The reason could be that in comparison with our patients, patients who underwent aortic surgery in the study by Welten et al. had more blood loss, which could lead to severe AKI and increase the risk of long-term mortality. In addition, the study of Bhatraju et al. found that when compared with no AKI patients, recovered AKI patients and non-recovered AKI patients have a worse long-term prognosis. This may be related to the endpoint (20). The endpoint of the study by Pavan K. Bhatraju et al. is a major adverse kidney event, defined as incidence or progressive CKD, long-term dialysis, or all-cause death.

At present, most of the studies that focused on the factors and mechanism of renal function recovery are aimed at AKI patients. Hickson et al. and Schmitt et al. found that baseline eGFR and aging are important factors affecting the recovery of renal function $(21,22)$. This is consistent with our results. We know that when compared with recovered CA-AKI and 

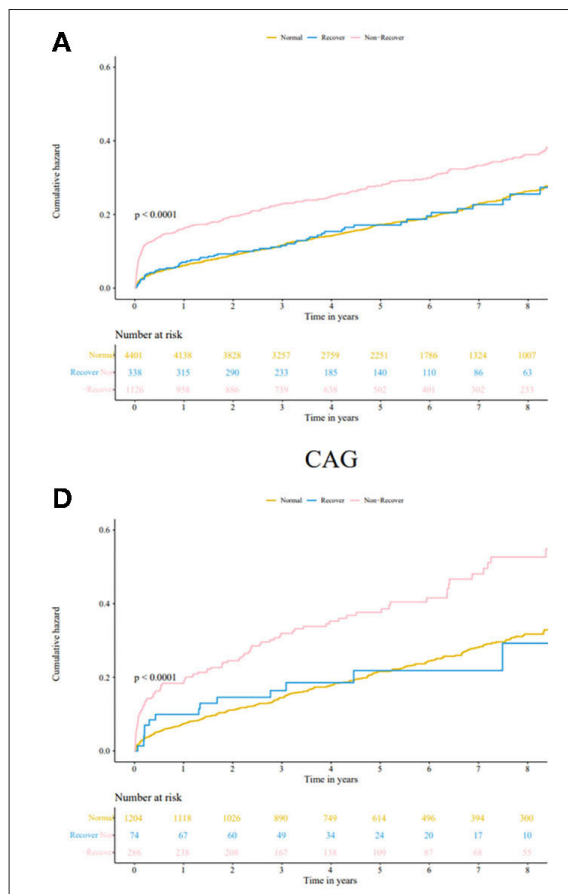

DM

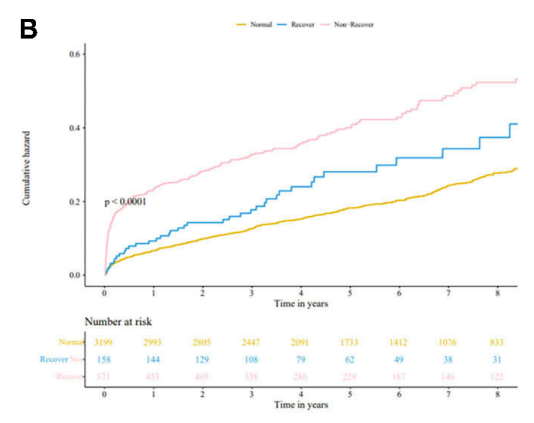

$\mathrm{CAD}$

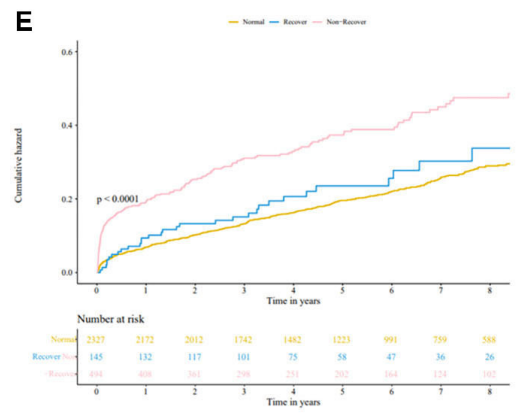

HT

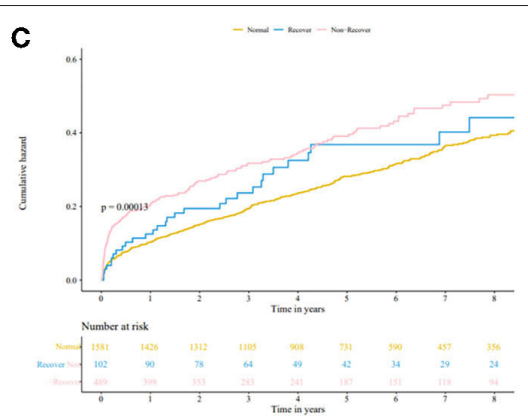

CKD

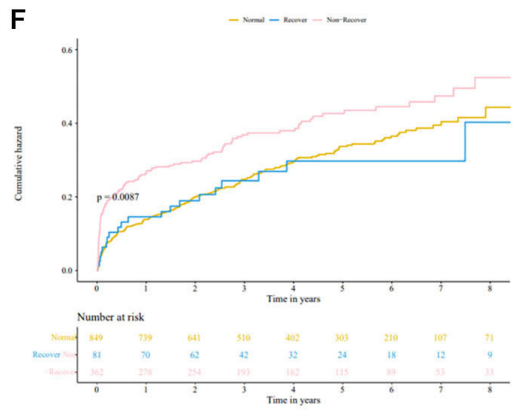

$\mathrm{HF}$

FIGURE 2 | Kaplan-Meier curves for the cumulative probability of mortality stratified according to no contrast-associated acute kidney injury (CA-AKI), recovered CA-AKI, and non-recovered CA-AKI. (A) coronary angiography patients; (B) coronary artery disease patients; (C) chronic kidney disease patients; (D) diabetes mellitus patients; (E) hypertension patients; (F) chronic heart failure patients.

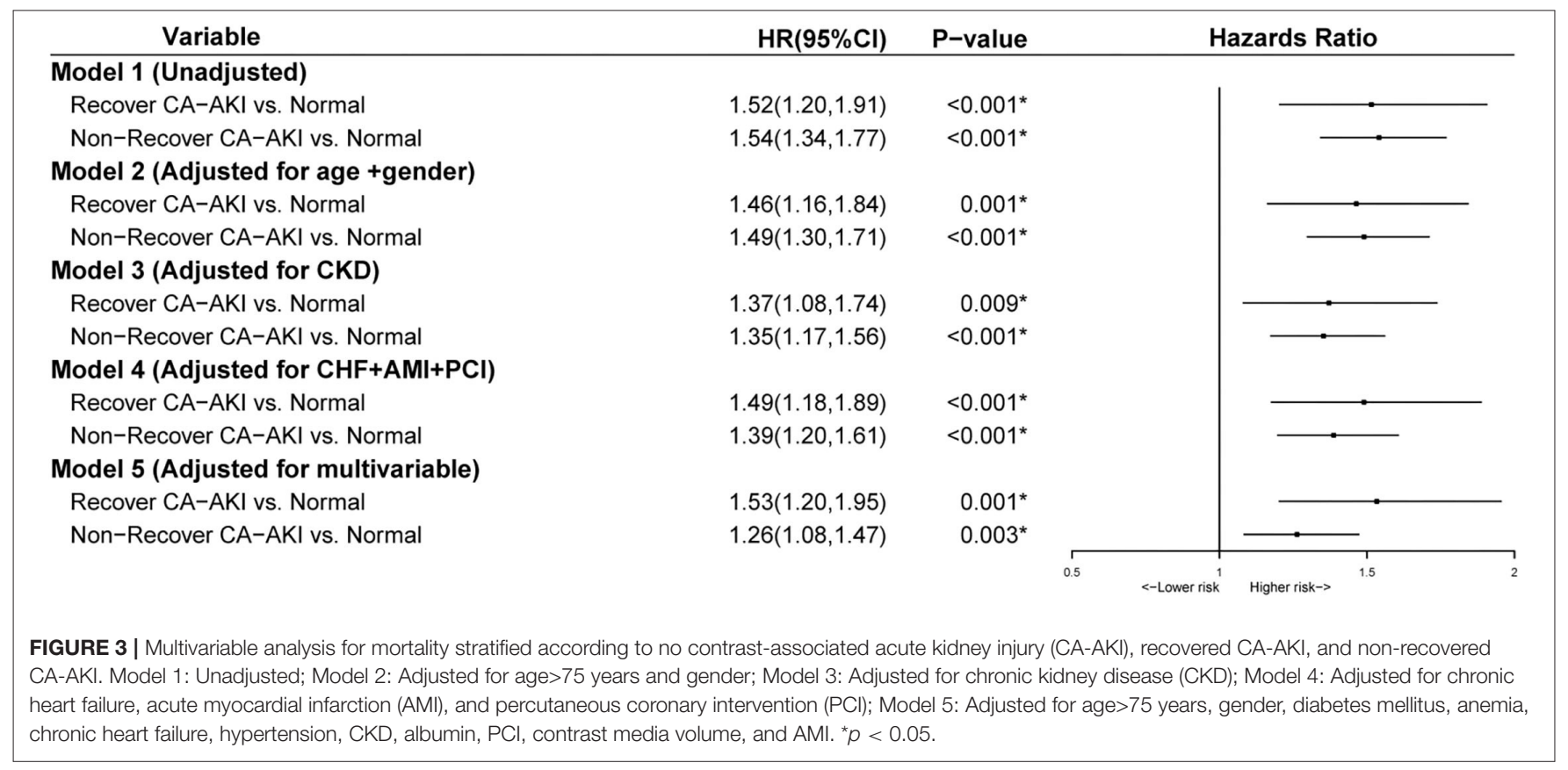

no CA-AKI patients, non-recovered CA-AKI patients have a higher incidence of chronic heart failure (Table 1). This is similar to the results of Hickson et al., which showed that patients with heart failure are more difficult to recover from kidney damage. Furthermore, for patients with AKI, who require renal replacement therapy, maintaining kidney perfusion, avoiding hypotension, and avoiding nephrotoxins and technique-related infection can help in the recovery of the kidney (23). Future 


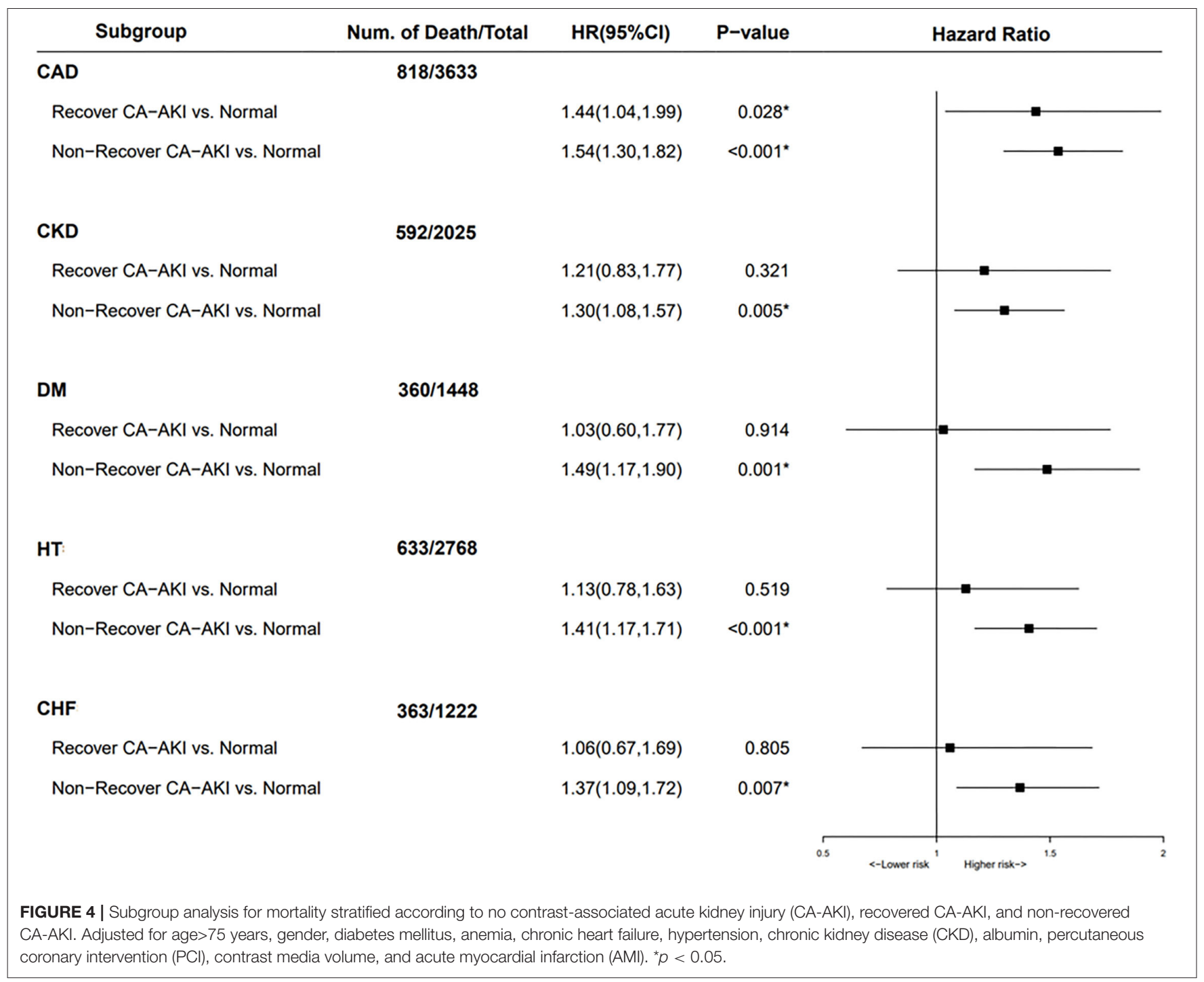

research needs to clarify the renal recovery mechanism of CA-AKI patients to reduce the burden of disease.

According to the subgroup analysis results, we confirmed that non-recovered CA-AKI was associated with an increased risk of long-term mortality in patients with complications, including CKD, diabetes mellitus, hypertension, and CHF. This further confirms our results and increases the generalizability of our results. Therefore, we should pay more attention to the prevention of non-recovered CA-AKI in the clinics to reduce its occurrence. At present, CA-AKI prevention mainly focuses on renal replacement therapy, drugs, and intravenous crystalloid (24). However, the benefits of preventive renal replacement therapy and most drugs have not been proven. Current guidelines recommend the use of isotonic saline to reduce the risk of CAAKI (25). In a meta-analysis of 124 studies, it was found that compared with saline, statins, xanthine, $\mathrm{N}$-acetylcysteine (NAC), and sodium bicarbonate could reduce the occurrence of CAAKI (26). It was also necessary to reduce the dose of contrast medium for high-risk patients. Future research should pay more attention to clarifying the mechanism of CA-AKI and explore more preventive measures for CA-AKI.

\section{Limitations}

Our research has several limitations. First, our study is a single-center retrospective analysis, which may lead to a lack of representativeness of our results. However, our study has a large sample size, and the results have been verified in multiple subgroups, which makes our results more credible. Second, long-term mortality is complex and multivariable. Due to the lack of other endpoint events, promotion of our results will be limited. Third, the definition of CA-AKI is diverse. In our research, CA-AKI was defined as an increase in $\mathrm{SCr}$ $\geq 50 \%$ or $\geq 0.3 \mathrm{mg} / \mathrm{d}$ from baseline within $72 \mathrm{~h}$ after the CAG. Fourth, we excluded patients who lacked SCr values at $72 \mathrm{~h}$ postoperatively, which resulted in the exclusion of $92 \%$ of the patients. Without the results of $\mathrm{SCr}$ at $72 \mathrm{~h}$ after surgery, we could not assess the recovery of renal function, and this in turn could lead to selection bias. However, we 
also performed a subgroup analysis, which makes our results more credible. Fifth, we were unable to obtain the patient's SCr during follow-up, which prevented us from assessing the long-term recovery of renal function after discharge. This has important implications for assessing whether a patient will develop $\mathrm{CKD}$, as it has important implications for assessing long-term mortality.

\section{CONCLUSION}

In conclusion, we found that non-recovered CA-AKI is associated with a worse prognosis. This means that we need to pay more attention to CA-AKI patients whose renal function has not recovered after CAG. Moreover, future research still needs to pay attention to the recovery mechanism of CA-AKI patients.

\section{DATA AVAILABILITY STATEMENT}

The raw data supporting the conclusions of this article will be made available by the authors, without undue reservation.

\section{ETHICS STATEMENT}

The studies involving human participants were reviewed and approved by Ethics Committee of Guangdong Provincial People's

\section{REFERENCES}

1. Chalikias G, Drosos I, Tziakas DN. Contrast-induced acute kidney injury: an update. Cardiovasc Drugs Ther. (2016) 302:21528. doi: 10.1007/s10557-015-6635-0

2. McCullough PA, Choi JP, Feghali GA, Schussler JM, Stoler RM, Vallabahn RC, et al. Contrast-induced acute kidney injury. J Am Coll Cardiol. (2016) 6813:1465-73. doi: 10.1016/j.jacc.2016.05.099

3. Rihal CS, Textor SC, Grill DE, Berger PB, Ting HH, Best PJ, et al. Incidence and prognostic importance of acute renal failure after percutaneous coronary intervention. Circulation. (2002) 10519:2259-64. doi: 10.1161/01.CIR.0000016043.87291.33

4. Weisbord SD, Chen H, Stone RA, Kip KE, Fine MJ, Saul MI, et al. Associations of increases in serum creatinine with mortality and length of hospital stay after coronary angiography. J Am Soc Nephrol. (2006) 1710:28717. doi: 10.1681/ASN.2006030301

5. Bartholomew BA, Harjai KJ, Dukkipati S, Boura JA, Yerkey MW, Glazier $S$, et al. Impact of nephropathy after percutaneous coronary intervention and a method for risk stratification. Am J Cardiol. (2004) 9312:15159. doi: 10.1016/j.amjcard.2004.03.008

6. Gruberg L, Mintz GS, Mehran R, Gangas G, Lansky AJ, Kent KM, et al. The prognostic implications of further renal function deterioration within $48 \mathrm{~h}$ of interventional coronary procedures in patients with preexistent chronic renal insufficiency. J Am Coll Cardiol. (2000) 365:15428. doi: 10.1016/S0735-1097(00)00917-7

7. Tervahartiala P, Kivisaari L, Kivisaari R, Vehmas T, Virtanen I. Structural changes in the renal proximal tubular cells induced by iodinated contrast media. Nephron. (1997) 761:96-102. doi: 10.1159/000190147

8. Guitterez NV, Diaz A, Timmis GC, O'Neill WW, Stevens MA, Sandberg KR, et al. Determinants of serum creatinine trajectory in acute contrast nephropathy. J Interv Cardiol. (2002) 155:349-54. doi: 10.1111/j.1540-8183.2002.tb 01067.x
Hospital (No. GDREC2019555H). The patients/participants provided their written informed consent to participate in this study.

\section{AUTHOR CONTRIBUTIONS}

DZ, ZL, BW, JL, NT, JC, YL, and JY contributed to the conception of the study. DZ, ZL, BW, JL, YL, JY, LL, GC, HL, MY, and SC contributed significantly to the data analysis and manuscript preparation. DZ, ZL, BW, JL, YL, and JY performed the data analyses and wrote the manuscript. DZ, ZL, BW, and JL contributed to the design and statistical analysis of this study. All authors have read and approved the manuscript.

\section{FUNDING}

The study was supported by the National Natural Science Foundation of China (Grant nos. 81670339 and 81970311), the Beijing Lisheng Cardiovascular Pilot Foundation (Grant no. LHJJ201612127), the Science and Technology Planning Project of Guangdong Province (Grant no. 2014B070706010), the Science and Technology Planning Project of Guangzhou (Grant no. 201704020124), Dengfeng Project in Guangdong Province (DFJH201919 and DFJH2020026), and Dongguan Social Science and Technology Development Key Project (202050715002176).

9. Briguori C, Quintavalle C, De Micco F, Condorelli G. Nephrotoxicity of contrast media and protective effects of acetylcysteine. Arch Toxicol. (2011) 853:165-73. doi: 10.1007/s00204-010-0626-5

10. Keaney JJ, Hannon CM, Murray PT. Contrast-induced acute kidney injury: how much contrast is safe? Nephrol Dial Transplant. (2013) 286:137683. doi: $10.1093 /$ ndt/gfs602

11. Romano G, Briguori C, Quintavalle C, Zanca C, Rivera NV, Colombo A, et al. Contrast agents and renal cell apoptosis. Eur Heart J. (2008) 2920:256976. doi: 10.1093/eurheartj/ehn197

12. Pannu N, James M, Hemmelgarn B, Klarenbach S, Alberta Kidney Disease N. Association between AKI, recovery of renal function, and long-term outcomes after hospital discharge. Clin J Am Soc Nephrol. (2013) 82:194202. doi: 10.2215/CJN.06480612

13. Welten GM, Schouten O, Chonchol M, Hoeks SE, Feringa HH, Bax JJ, et al. Temporary worsening of renal function after aortic surgery is associated with higher long-term mortality. Am J Kidney Dis. (2007) 502:21928. doi: 10.1053/j.ajkd.2007.04.002

14. Lee BJ, Hsu CY, Parikh RV, Leong TK, Tan TC, Walia S, et al. Non-recovery from dialysis-requiring acute kidney injury and short-term mortality and cardiovascular risk: a cohort study. BMC Nephrol. (2018) 191:134. doi: 10.1186/s12882-018-0924-3

15. (KDIGO). KDIGO clinical practice guideline for acute kidney injury. Kidney Int Suppl. (2012) 2:1-138.

16. National Kidney F. K/DOQI clinical practice guidelines for chronic kidney disease: evaluation, classification, and stratification. Am J Kidney Dis. (2002) 392(Suppl. 1):S1-266.

17. Manjunath G, Tighiouart H, Ibrahim H, MacLeod B, Salem DN, Griffith $\mathrm{JL}$, et al. Level of kidney function as a risk factor for atherosclerotic cardiovascular outcomes in the community. J Am Coll Cardiol. (2003) 411:4755. doi: 10.1016/S0735-1097(02)02663-3

18. Nutritional anaemias. Report of a WHO scientific group. World Health Organ Tech Rep Ser. (1968) 405:5-37. 
19. Wehbe E, Duncan AE, Dar G, Budev M, Stephany B. Recovery from AKI and short- and long-term outcomes after lung transplantation. Clin J Am Soc Nephrol. (2013) 81:19-25. doi: 10.2215/CJN.04800512

20. Bhatraju PK, Zelnick LR, Chinchilli VM, Moledina DG, Coca SG, Parikh $\mathrm{CR}$, et al. Association between early recovery of kidney function after acute kidney injury and long-term clinical outcomes. JAMA Netw Open. (2020) 34:e202682. doi: 10.1001/jamanetworkopen.2020.2682

21. Hickson LJ, Chaudhary S, Williams AW, Dillon JJ, Norby SM, Gregoire JR, et al. Predictors of outpatient kidney function recovery among patients who initiate hemodialysis in the hospital. Am J Kidney Dis. (2015) 654:592602. doi: 10.1053/j.ajkd.2014.10.015

22. Schmitt R, Coca S, Kanbay M, Tinetti ME, Cantley LG, Parikh CR. Recovery of kidney function after acute kidney injury in the elderly: a systematic review and meta-analysis. Am J Kidney Dis. (2008) 522:26271. doi: 10.1053/j.ajkd.2008.03.005

23. Palevsky PM, Baldwin I, Davenport A, Goldstein S, Paganini E. Renal replacement therapy and the kidney: minimizing the impact of renal replacement therapy on recovery of acute renal failure. Curr Opin Crit Care. (2005) 116:548-54. doi: 10.1097/01.ccx.0000179936.21895.a3

24. Mehran R, Dangas GD, Weisbord SD. Contrast-associated acute kidney injury. N Engl J Med. (2019) 38022:2146-55. doi: 10.1056/NEJMra1805256

25. Neumann FJ, Sousa-Uva M, Ahlsson A, Alfonso F, Banning AP, Benedetto U, et al. 2018 ESC/EACTS guidelines on myocardial revascularization. Eur Heart J. (2019) 402:87-165. doi: 10.15829/1560-4071-2019-8-1 51-226
26. Giacoppo D, Gargiulo G, Buccheri S, Aruta P, Byrne RA, Cassese $S$, et al. Preventive strategies for contrast-induced acute kidney injury in patients undergoing percutaneous coronary procedures: evidence from a hierarchical bayesian network meta-analysis of 124 trials and 28240 patients. Circ Cardiovasc Interv. (2017) 10:e004383. doi: 10.1161/CIRCINTERVENTIONS.116.004383

Conflict of Interest: The authors declare that the research was conducted in the absence of any commercial or financial relationships that could be construed as a potential conflict of interest.

Publisher's Note: All claims expressed in this article are solely those of the authors and do not necessarily represent those of their affiliated organizations, or those of the publisher, the editors and the reviewers. Any product that may be evaluated in this article, or claim that may be made by its manufacturer, is not guaranteed or endorsed by the publisher.

Copyright (C) 2022 Zhou, Lun, Wang, Liu, Liu, Chen, Ying, Li, Chen, Tan, Chen, Liu and Ye. This is an open-access article distributed under the terms of the Creative Commons Attribution License (CC BY). The use, distribution or reproduction in other forums is permitted, provided the original author(s) and the copyright owner(s) are credited and that the original publication in this journal is cited, in accordance with accepted academic practice. No use, distribution or reproduction is permitted which does not comply with these terms. 